\title{
Next Steps for Compressively Sensed Video in Transmission Electron Microscopy
}

\author{
B. W. Reed ${ }^{1}$, N. Moghadam ${ }^{1}$, R. S. Bloom ${ }^{1}$, S. T. Park ${ }^{1}$, and D. J. Masiel ${ }^{1}$ \\ 1. Integrated Dynamic Electron Solutions, Inc., Pleasanton, CA, USA.
}

Compressive sensing (CS) is a fast-emerging approach for improving data throughput by taking advantage of the inherent compressibility of real world data - the same kind of compressibility that allows millions of users to stream full-screen video without breaking the internet. Transmission electron microscope (TEM) users regularly collect multi-GB data sets and then use aggressive data reduction strategies to draw out the tiny residual of Shannon entropy that answers the question they wanted to ask. Much of the raw-byte-count information in an acquisition is random, redundant, or irrelevant, yet its collection still represents substantial cost in terms of instrumentation bandwidth and sample damage.

CS works by operating equipment in a nonstandard way such that data compression is performed in the analog domain, before the analog-to-digital convertor (ADC) bottleneck. Assuming we are limited primarily by this bottleneck, a naïve approach that gives equal attention to every pixel and every point in time, faithfully capturing every single one, is only optimal if we expect to be measuring perfectly uncorrelated white noise. CS recognizes that one always has a priori information, that real images almost never look like pure white noise, and that this prior knowledge should inform the strategy for collecting data if one wants to open up the ADC bottleneck. This concept is well developed in the mathematical literature (for a recent review, see [1]).

In the past, we have presented proof-of-principle results for CS video acquisition in a TEM that can produce $\mathrm{kHz}$-scale video using a conventional Hz-scale camera, with 50-100 output frames per input frame [2]. The system operates through use of a fast, arbitrarily programmable, zero-hysteresis postsample deflection unit that sends images to multiple regions on a camera (Figure 1). Now one would like to go beyond proof of principle and bring this and other CS techniques into the hands of ordinary users, including those who know little or nothing about CS, while still ensuring quality, reliable results. Towards this end, we advocate several system design principles:

First, the technique needs to be easy, with natural integration into familiar workflows. We have developed the deflector system such that it installs and operates much like other familiar hardware (e.g. STEM detectors and side-mount cameras). Software and hardware integration allow automatic timing control, synchronization with beam blankers and in situ sample drives, and automatic data processing to allow distortion corrections and camera noise calibrations in real time. Calibration procedures are deliberately made analogous to already-familiar procedures (Figure 2).

Second, data analysis needs to be transparent and user-friendly, with calibrated errors providing confidence in the result. It can be hard to trust complicated analysis approaches that, in a series of semiopaque steps, calculate backwards from a measurement to create some idealized ground truth model. It is both more familiar and more accepted to calculate forwards, from ground truth to the detector, with calibrated models for distortion, noise, imperfections, etc. This enables fully calibrated $\chi^{2}$ analysis, i.e. quantitatively assessing the degree to which a ground truth model is consistent with the measurement. A CS reconstruction is closely related to a familiar concept — data/model fitting with quantitative error propagation — but with a sophisticated model for prior knowledge about the ground truth. Features that 
are robust with respect to reasonable changes in the model can then be reasonably trusted, just as with more conventional and familiar analysis techniques.

Third, the analysis tools need to be made widely available and easy to understand, which is best achieved by (1) Modeling the reconstruction analysis on familiar image-processing tasks and making them available in standard TEM image acquisition/analysis software, (2) Developing the algorithms so they work equally well on CS and conventional data, and (3) Giving users full control over the analysis parameters and direct access to their own raw data in well-documented standard formats [3].

\section{References:}

[1] M. Rani, S. B. Dhok, and R. B. Deshmukh, IEEE Access PP, issue 99, DOI:10.1109/ACCESS.2018.2793851 (2018).

[2] B. W. Reed et al., Microsc. Microanal. 23 (Suppl 1) (2017), p. 84.

[3] This material is based upon work supported by the U.S. Department of Energy, Office of Science, Office of Basic Energy Sciences, under Award Number DE-SC0013104.

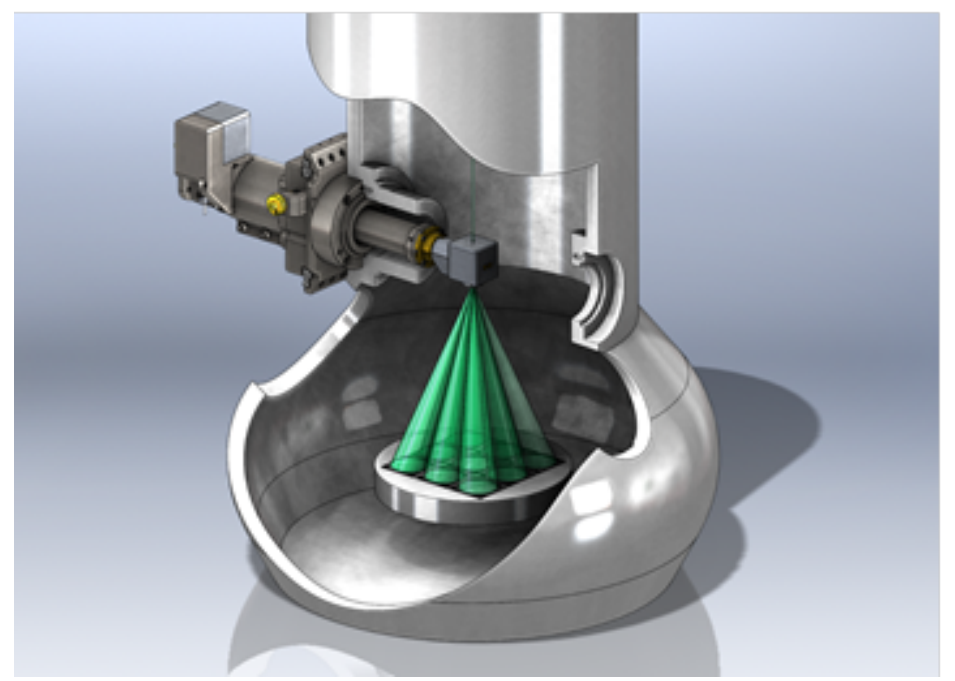

Figure 1. The deflector is inserted into a standard detector port. A fast switching circuit controlled by an arbitrarily programmable digital pattern generator sends images to multiple subregions on a camera, rapidly jumping back and forth from one image position to another, in a way that encodes up to 100 frames of video in one exposure. The pattern generator automatically synchronizes the deflector with the camera and beam blanker while providing precise downstream triggering of in situ drives, fast blankers, etc. This enables multiple operating modes besides just CS video.

Figure 2. An example of the automated alignment routine, which collects one image from each deflected position and performs robust feature matching to automatically produce a map of all distortions and offsets. Deflector distortion is repeatable to $\sim 1$-pixel precision and thus can be reliably corrected. Once calibrated, this can be performed in the background and the results handed to the user, much like ordinary gain/flat-field camera corrections. Users will also be able to easily access the raw data.

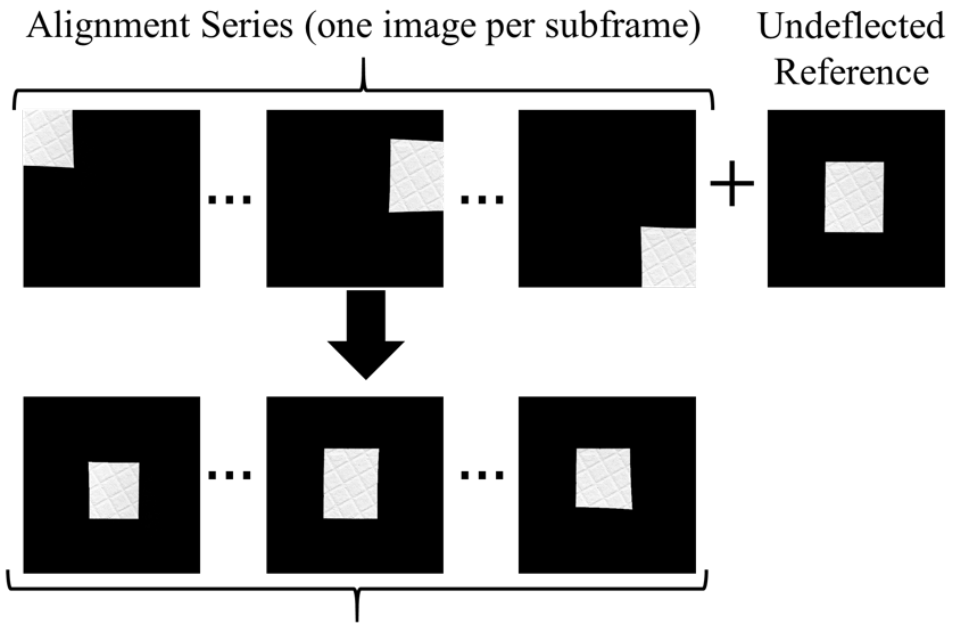

Aligned spots (corrected distortion and offsets) 